\title{
Multi-access edge computing: open issues, challenges and future perspectives
}

\author{
Sonia Shahzadi', Muddesar lqbal², Tasos Dagiuklas ${ }^{2 *}$ and Zia UI Qayyum³
}

\begin{abstract}
Latency minimization is a pivotal aspect in provision of real time services while adhering to Quality of Experience (QoE) parameters for assuring spectral efficiency. Edge Cloud Computing, being a potential research dimension in the realm of $5 \mathrm{G}$ networks, targets to enhance the network efficiency by harnessing effectiveness of both cloud computing and mobile devices in user's proximity. Keeping in view the far ranging impact of Edge Cloud Computing in future mobile generations, a comprehensive review of the prevalent Edge Cloud Computing frameworks and approaches is presented with a detailed comparison of its classifications through various QoS metrics (pertinent to network performance and overheads associated with deployment/migration). Considering the knowledge accumulated, procedures analysed and theories discussed, the paper provides a comprehensive overview on sate-of-the-art and future research directions for multi-access mobile edge computing.
\end{abstract}

Keywords: Cloud computing, Cloudlets, Edge cloud, Fog computing, Internet of things, Mobile cloud computing

\section{Introduction}

The forthcoming emergence of Internet over Everything is driven by the evolution of $5 \mathrm{G}$ communication, rapid growth of cloud, social media, and mobile computing, the use of Data Science to generate smart analytics value. This evolution brings to the forefront new type of communications such as Machine to Machine and Person to Machine [1]. According to RCR wireless, more than 50 Billion Internet of Things (IoT) devices will be interconnected by 2020 [2]. In this new environment, there is a need to manage, process and store the huge amount of data generated at the network edges. Cloud computing frees the enterprise and the end users from many details. As an effect, computational and network overhead at central cloud increases. This creates problems with real-time applications where latency is a crucial factor. Edge Cloud (EdgeC) Computing has been introduced to reduce network stress (i.e. latency) by shifting resources at the edge of network to proximity of mobile users and IoT while providing services and seamlessly processing the contents. As it implies, the idea of EdgeC has emanated from Cloud Computing (CC) leading towards to Mobile Cloud Computing (MCC). It

\footnotetext{
* Correspondence: tdagiuklas@lsbu.ac.uk

${ }^{2}$ London South Bank University, London, UK

Full list of author information is available at the end of the article
}

offers cloud resources at the edge of network with low latency and high bandwidth. Mobile Edge Computing (MEC) started to gain attention of research community in last few years with preliminary research contributions so far such as: standardization of some key interfaces for mobile edge computing [3], building super-short applications requiring a low response time and latency [4-6] and modeling hyper-scale datacentres with micro datacentres at the edge of networks [7]. Executing the computing-intensive applications consume lots of power at the mobile device. The advances in EdgeC have made it possible to provide infrastructure, platform, and software as a service for the end-users from any computer with a fixed or wireless Internet connection. EdgeC can extend such services to mobile devices. Since there are several billions of mobile subscribers world-wide, EdgeC has the potential to have far-reaching impacts in the wireless industry and in our society. The delivery of demanding applications (e.g. streaming, augmented reality, on-line gaming etc) to/from the cloud to the mobile users relies on wireless networks (e.g., WiFi, 3G, 4G, 5G etc) for data and control between the cloud and mobile devices. Compared with fixed networks, wireless networks have limited bandwidth, latency due to network congestion, and connectivity. Moreover, under the presence of more mobile devices, the bandwidth available to 
each device will be further reduced, and network latency can go up and response time for mobile users can be higher. The main objectives of this paper are to present use case scenarios associated with Edge Cloud Computing, describe the latest advances in different standardization fora related to Edge Cloud Computing, discuss future research challenges. In remainder of this paper, section "Motivation" describes the application scenarios and motivation; section "Edge Cloud Computing Standardization and Fora" describes different approaches for Edge Cloud computing and section "Comparative Analysis of Existing Frameworks" describes the classification and comparison of existing frameworks. The section "Open issues and challenges" presents the open research challenges and issues and paper is finally concluded in section "Conclusion".

\section{Motivation}

As an effect, the initial objective of EdgeC is to adapt cloud computing to the mobile environment in anywhere and anytime manner, where data are stored and processed outside mobile devices $[8,9]$. Some of the most critical issues related to $\mathrm{EdgeC}$ include: network latency and limited network bandwidth and user mobility. Despite the advances in smartphones, they still have limited processing capability and limited battery life, especially with the growing demand for energy-hungry applications, such as video streaming and 3D gaming. In Ref. [10] described MEC as an emerging paradigm that provides computing, storage and networking resources within the edge of mobile Radio Access Network (RAN). The preparation for deployment of $5 \mathrm{G}$ network and tactile Internet sparked conversations about issues that need to be solved to increase the QoE of applications based on this platform. These applications require low latency and real-time data to effectively utilize its functionalities. Research done by [11] observed that the existing cloud infrastructure cannot resolve this issue. In Ref. [12] explained the key issue mobile edge computing aims to solve is to reduce the network bandwidth and latency in other to improve QoE. This would be done by bringing cloud infrastructure closer to the user. In Ref. [13] demonstrated that deploying cloudlets in close proximity with the end user improves the execution of latency-critical applications.

The trend of pushing cloud computing to the edge of mobile networks are expected to continue to accelerate in years to come. According to [10] the challenges and open issues associated with MEC includes data interoperability, resource management, and orchestration, service discovery and security.

This section present scenarios are highlighted in the following section where MEC can be beneficial in terms of performance improvements [5].

\section{Augmented Reality (AR)}

Augmented reality (AR) merges the view of real world and computer generated sensory inputs such as graphics, GPS data, sound and video [5]. AR allows the user to see the real world, with virtual objects superimposed upon or composited with the real world so that the information about the surrounding real world of the user becomes interactive and digitally manipulable. EdgeC can be used for generation of rendering. Required processing can be performed on EdgeC instead of the main server due to requirement of high processing speed and low latency.

\section{Connected vehicles}

The number of connected vehicles has been increased to support Vehicle-to-everything (V2X) communication, e.g. inform vehicles about road conditions through image/ video analysis route prediction, collision warning applications such as safety, infotainment and communication or any other information that may affect the vehicle. Furthermore, the use of Roadside Units is intended to increase efficiency, and convenience of the V2X applications [14]. As the number of connected vehicles increases and use cases evolve, the volume of data will continue to increase along with the need to minimize latency and optimize QoE. EdgeC can be very useful to push V2X applications, data, and services from central cloud to the edge of network (e.g. Roadside units), this would help in bringing data and analytics applications closer to the vehicles at the roadside units, enabling applications acceleration over the vehicles [15]. The Mobile Edge Computing application can operate as a highly distributed roadside unit to support vehicle-to-everything (V2X) communication. Thus helps in sending the useful information to the nearby cars without any delay. This Instant communication can help drivers to react in timely fashion in order to avoid accidents and improve road safety.

\section{Internet of Things (IoT)}

IoT is a network that connects physical devices, sensors, vehicles and everyday electronic objects embedded with software, actuators and sensors to collect and exchange data, but also goes beyond this to include connections and networking between transport services, community services and much more of societies infrastructure [16]. The IoT is the latest technology and it is as important as the Internet. It is a network that connects all things to the Internet for exchanging information and communication through devices with agreed protocols by identifying, locating, monitoring and managing things [17]. In other words, the Internet is no longer bound by the desktop, but goes out into the world of other things [16]. The enormous amounts of data generated by this process would be best stored on a cloud. Moving IoT 
application data to the cloud can reduce the cost and complexity that relates to hardware management [18]. There is a need to aggregate various IoT device messages using mobile cloud computing closer to the device users to improve latency and response time. Various devices are connected over different forms of connectivity, such as 3G, 4G, 5G, Wi-Fi or other radio technologies [19]. In general, the messages are small, encrypted and come in different forms of protocols (e.g. MQTT, CoAP etc). There is a need for a low latency aggregation point to manage the various protocols, distribution of messages and for the processing of analytics from data collected from different IoT deployments [20]. The EdgeC server provides the capability to resolve these challenges.

\section{Edge cloud media optimization}

The distributed Edge Clouds have been designed and developed to support the media services across heterogeneous wireless and converged networks. The EdgeC provides support to the immersive applications to handle challenges such as user mobility and scarce network resources. It also helps in developing cloud-based workflow management for media applications, intelligently serving the end users through the available communication capacity and end-user device capabilities. This necessitates carrying media related functionalities such as rate adaptation/transcoding, rendering and caching as shown in Fig. 1.

This use case aims to optimize QoE for video applications over radio access network. This can be accomplished by estimating throughput at the radio downlink interface from radio analytics information. EdgeC can be used to enhance QoE for the users by to adopting a video using application-level coding (e.g. transcoding, rate control) to matches the estimated capacity at the radio downlink [21].

The adoption of Edge Cloud could be adopted by network operators either at the access networks (e.g. evolved Node B (eNB), Wireless APs) or at the aggregation points that are interconnected to the core networks via backhauling. A major challenge is that the user mobility may affect the entire operation when he/she is associated in an AP where the network operator has not deployed. In this case, the operator may seek for on-the-fly computing resources by requesting the available computing resources in an on-demand fashion from third parties. Such third parties could be any foreign cloud provider with enough/available computing resources that could be 'rented' on demand by the network operator. This necessitates the establishment of an agreement between the involved parties through a federation scheme. An immense volume of resources possibly with different specifications need to be managed under a unified and federated framework in terms of physical nodes and their accompanying physical and virtual resources. Once the requested computing resources are transferred to the network operator, another task is to manage computing resources. Due to mobility, the user may be associated (using handover management) to an area where neither the AP nor the aggregated node has EdgeC resources. In this case, the computing resources that will be requested by the third party can be established. After this establishment, cloud

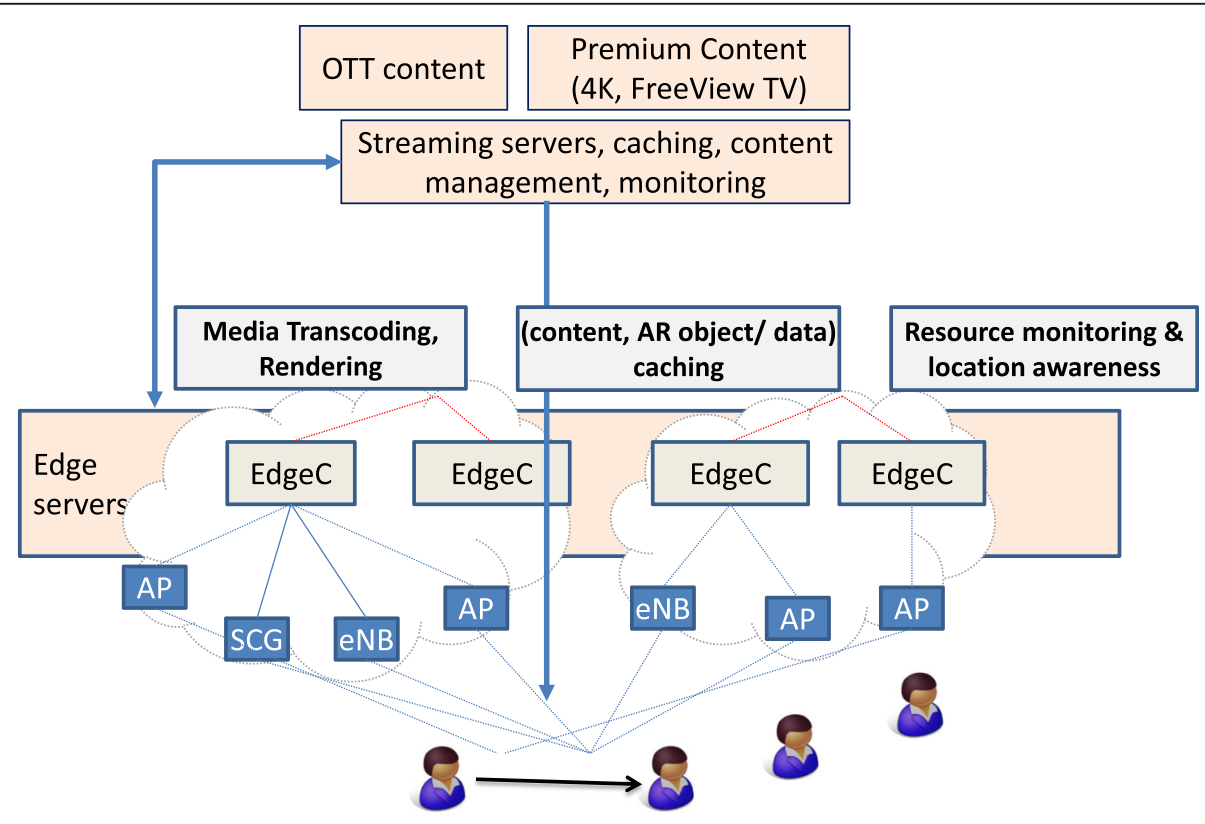

Fig. 1 Edge cloud media optimization 
computing resources must be transferred from the old EdgeC to the new one. This scenario is illustrated in the Fig. 2.

\section{Edge cloud computing standardization and Fora}

In this section, some relevant approaches presenting similar concept are comprehensively elaborated. These approaches are broadly categorized into (1) MEC based approaches (2) Cloudlets based approaches and (3) Open Fog Consortium.

\section{MEC approach}

ETSI introduced Mobile Edge Computing (MEC) in ETSI [3] which is designed to push resources closer to the radio access networks in 4G and 5G. It brings cloudcomputing capabilities and IT service environment at the edge of the mobile network. This environment is characterized by ultra-low latency and high bandwidth as well as real-time access to radio network information that can be leveraged by applications. Since 2017, the ETSI MEC industry group has renamed "Mobile Edge Computing" to "Multi-Access Edge Computing" to better reflect the growing interest in MEC from noncellular operators. MEC has the aim to reduce network stress by moving resources from cloud to mobile edge $[22,23]$. Fully virtualized MEC infrastructure is proposed in [23]. A distributed computation offloading algorithm is presented in [24]. SEcS (Scalable Edge computing Services) framework is presented to build and deploy Edge computing Services to address the challenge of scalability, high availability, fault tolerance and robustness [25]. Multi-access MEC architecture is designed to addresses latency and bandwidth issues for the video analytics location services, Internet-of-Things (IoT), augmented reality, optimized local content distribution and data caching and many other use cases and application scenarios for Smart Cities, Healthcare, Disaster Management and Smart farming. A scheme is proposed in [26] to deal with unpredictability of computation availability at the edge, where task execution performed on idle edge resources. In [27], it argues that autonomic computing techniques are fundamental element for dynamic management of edge servers. MEC architecture is proposed in [28] to reduce latency. To migrate running application from VM or container for mobile edge cloud environment, a layered solution is proposed in [29]. A WiCloud is proposed in [30] that is based on NFV/SDN concepts to provide edge networking. Some frameworks are given in [31] for mobile application execution in MCC and their comparative study are also given. Seamless application execution frameworks in MCC are also highlighted in [32] with detailed comparisons and analysis.

\section{Cloudlets based approach}

The term "cloudlet" was coined by researcher at Carnegie Mellon University, where its prototype is developed as part of a research project [33]. The Cloudlets are designed to support applications for mobile devices those are resource hungry and interactive e.g. Augmented reality applications, Cloud games, Wearable cognitive assistance system Google Glass, Apple Siri and Google Now and many other applications those require offloading of resource intensive task from cloud to the mobile device to achieve the required quality of experience. This helps in reducing communication latency and perform faster execution for application intends to perform resource intensive tasks. The main motivation of cloudlet comes from the Internet community to handle the resource constraint on the mobile devices.

A cloudlet represents the middle tier of a 3-tier hierarchy i.e. Mobile device, Cloudlet, Cloud. Cloudlets can

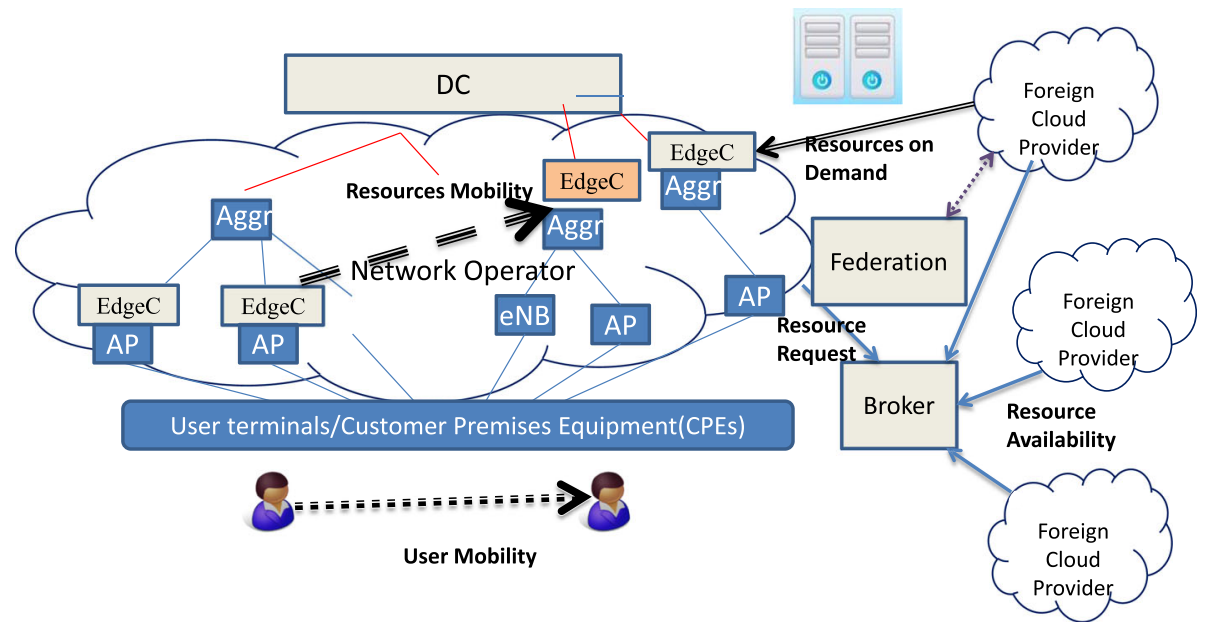

Fig. 2 Distributed cloud federation and service brokerage model 
be considered as a local data centre in a box to enable localized cloud services, offer high performance and faster access to cloud resources by multiple users simultaneously [34]. Moreover, it deals with large WAN latency, less bandwidth, and high utilization cost issues [34]. Cloudlet, through the interest of key industrial players (e.g. Nokia, Intel, Vodafone) have formed the open source banner of Open Edge Computing (OEC) Initiative. OEC has offered cloudlets open-source code APIs as an extension to OpenStack to promote cloudlet as an enabling technology [35]. The main goal is to, engage with wider IT and Telecoms industry to Synchronize the work with other efforts includes ETSI ISG MEC and OPNFV. The cloudlet pioneering Elijah project at Carnegie Mellon University has been extended to OpenStack++: to provide a cloudlet library based on a modified QEMU with integration into the OpenStack platform. A mesh cloud architecture is proposed in [34], which is composed of cloudlet, Internet cloud and wireless mesh networks. An experimental framework is designed in [36], in which private cloudlet and wireless mesh network is implemented. It is capable of establishing and maintaining mesh connectivity among multiple nodes automatically and is featured with adaptivity and self-recovery in case of network failures.

Cloudlet architecture presented in [37], manages applications at the component level. Cloudlet based MCC system is introduced in [38] for reduction of power consumption and network delay. A Performance Enhancement Framework for Cloudlet (PEFC) is proposed for MCC [39] to improve the cloudlet performance. Centralized cloudlet architecture is proposed in [40]. In [37], a new cloudlet architecture is proposed where applications are dealt on a component level where components are distributed among dynamic cloudlets letting users to join and leave cloudlets at runtime. However, cloudlet performance relies upon user mobility [41]. In [42], two migration models have been compared cloudlet network design i.e. VM bulk migration and VM live migration. In order to access discoverable cloudlet server for mobile users for resource provision and services on demand, a cloudlet system is proposed in [43]. These cloudlets may be deployed at various public places where users can connect cloudlet through a mobile network provider [43].

Another cloudlet based system is proposed in [39], with focus on performance improvement in mobile cloud computing. Cloudlet is installed along with AP to allow mobile devices to access it. These mobile devices connect to nearby cloudlet using Wi-Fi [38]. To minimize, delay and power costs of mobile user, cloudlet infrastructure is proposed in [44]. In [45], an energy management approach is introduced for mobile/pocket cloudlet. Researchers and Marine Corps are working together on the concept of "tactical cloudlet" to implement distributed cloud computing concept in a remote and mobile battlefield scenario, especially in a more hostile environments e.g. during a war mission or disaster recovery where the requirements for communication changes quickly and requires higher power for computing [46]. Tactical cloudlets are proposed in [47] to support tactical edge and cyber-foraging where resource intensive tasks are offloaded to cloudlets. A strategy is proposed in [48], to reduce multi-resource allocation problem between cloudlet and mobile devices that will enhance Quality of Service (QoS).

\section{Open fog consortium}

Fog Computing is a concept introduced by Cisco in 2011 to meet the demands from different segments of Internet of Things (IoT), Internet of Everything (IoE) or Internet of Me (IoM) start to take off, e.g., consumer, wearable, industrial, enterprise, automobile, healthcare, building, energy. Classical cloud computing paradigm can hardly satisfy low latency, mobility support and location awareness. To address these problem, fog computing paradigm is introduced which improves quality of services (QoS) for real time applications and streaming, provides low latency and location awareness [49] in the field of wireless sensor networks, industrial automation and transportation systems. The main motivation is to alleviate the disadvantages of cloud computing: Long WAN latencies is a big obstacle in the critical path of user interaction and can deteriorate usability, traffic to central cloud increases computational and network overhead at central cloud. Fog computing introduces decentralized computing infrastructures so that computing resources and applications services are distributed in the most logical, efficient places, at any point along the continuum from the data source to the cloud. The main emphasis of the open fog consortium is to define a system-level horizontal architecture that distributes resources and services of computing, storage, control and networking anywhere along the continuum from Cloud to Things [50]. It's put data close to the end user [51] which reduce latency and improve QoS [52]. It also enables localization, context awareness and mobility support [49]. The Decoy Information techniques are used to detect malicious attacks those cannot be addressed using traditional security measures such an attack by an insider by seeding data into a system which appears genuine but actually it is fact spurious. Using Decoy information technology, you can implement security in fog computing [52]. Fog provide high quality streaming through access points and proxies to mobile nodes including moving vehicles [53]. It is suitable for those applications that require predictable and low latency such as video conferencing and gaming [54]. Fog architecture is given in [55] and radio access network (F-RAN) based fog is presented in [56]. 
In fog computing, cloud resources such as compute and storage etc. are migrating to the edge of the network where routers themselves may become the virtualized infrastructure [8] and services can be hosted at end devices such as set-top-boxes or access points [49]. In addition, multiple heterogeneous decentralized and ubiquitous devices communicate and cooperate with each other and can perform processing and storage tasks using network without the interference of third-parties [8]. It provides highly virtualized platform that offers storage, computing and networking services between the main cloud data centers and end devices [9]. It supports multiple services and applications where low latency is required. Fog/ edge nodes have sufficient computing power to facilitate user's task that are received from their end devices. This edge computing concept is introduced within cloud to reduce end-to-end response time between multiple devices. Although cloud computing provides lot of benefits to users in terms of cost reduction, system administrative tasks eliminations, flexibility increase, and improve reliability etc. but it also suffers some limitations including unpredictable network latencies and security issues etc. To overcome these limitations, fog computing is introduced where cloud system is located at the edge of network [57]. Fog computing will be helpful for emerging network paradigm which requires faster processing with less delay [57]. It is able to provide high quality streaming to mobile users through access points or proxies [53]. It is suitable for video streaming, gaming and augmented reality where low latency is required [53]. Fog computing not only reduces latency but also improves the QoS [51]. In fog computing paradigm, data is distributed and moved closer to the end-user and also support for data streaming and mobile computing [52]. Fog is considered to address services and applications that not well fit in cloud, e.g. video conferencing and gaming applications that need predictable and low latency, fast mobile applications, smart grid and smart traffic light system etc. [54]. In short, the aim of fog computing is to place cloud resources, close to mobile users [55]. A FSDN is proposed in [58], which combines the Software Defined Networking (SDN) and Fog computing.

ETSI-MEC consortium is developed to unite the IT Cloud and Telecommunication industry on MEC standards to providing IT and cloud-computing capabilities within the RAN through Mobile Orchestrator APIs for provisioning and monitoring virtual resources, targeting especially network function visualizations. Considering the overlapping interests in MEC and Cloudlets, a few on the other hand, Cloudlet/OEC have been motivated by the Internet community to optimize Internet demanding applications over resource-constrained mobile devices. Fog computing is mainly driven by IoT and the need for data processing and interoperability at the edge. All these three approaches presented have been compared in terms of different quality parameters in [35] are presented in Table 1.

\section{Comparative analysis of existing frameworks}

We have compared existing frameworks on the basis of various properties. Caching is used to store data locally to reduce the delay [32]. In case of cloudlet, it improves the latency by minimizing delay. Mirroring is also used to cache data at the mirror during uploading and downloading which also reduce the delay [59]. It reduces the operational overhead and optimizes response time but it increases the cloud storage cost [32]. Parallel execution improves the execution time of an application but it increases power consumption and hardware cost [32]. Preinstallations improves runtime data transmission but it increases the cloud resource consumption and maintenance overhead [32]. The optimize VM migration enables migration for only relevant applications instead of whole VM migration which reduces transfer overhead and improves the transmission time [32]. Fault tolerance provides a transparent mechanism to failure detection which requires a continuous monitoring but can create high complexity [32]. The reduction in the number of hop distance results in improved latency, jitter and response time. CloneCloud, a flexible application partitioner is proposed in [60]. It automatically distributes the computation tasks from single mobile device to multiple machines. It significantly improves task processing and reduce the energy of smart mobile devices. Although it provides optimal execution time for computation environment but it increases the data transfer overhead on multiple machines. A framework is proposed in [61], to perform the optimal dynamic partitioning and execution of applications. It provides high performance with low operational cost but it also increases the data transfer overhead. Another solution is proposed in [62], for dynamic adaptive deployment of applications to enhance the quality of service. It offers optimal deployment of applications but it depends on nearby servers. Hyrax, a platform to support mobile devices is derived from MapReduce [63] and provide infrastructure for mobile computing. It improves utilization of mobile resource but execution of MapReduce jobs on mobile phones results in high overhead for devices with limited resources. The work presented in [64] offers infrastructure deployment through CloneCloud architecture for smartphones applications to boost the mobile applications via cloning by using multiple computing platforms. It overcomes the limitations of mobile resources and the clone can be used as a recovery processing but it increases computation transformation overhead. The work presented in [65] provides highly dynamic and energy saving 
Table 1 Comparison of cloudlets, fog and MEC approaches [35]

\begin{tabular}{llll}
\hline Properties & Cloudlets based approach & Open fog computing & MEC approach \\
\hline Reduce Latency & $Y$ & $Y$ & $Y$ \\
Reduce Jitter & $Y$ & $Y$ & $Y$ \\
Multi-Tenancy & $Y$ & $Y$ & $Y$ \\
With virtual laaS platform? & $Y$ & $Y$ & $Y$ \\
Co-Location & $Y$ & $Y$ & $Y$ \\
Geographical Distributed & $Y$ & $Y$ & $Y$ \\
Mobility Support & $Y$ & $Y$ & $Y$ \\
Inspired from & Tactile Internet & loT & Mobile World \\
Extended from Cloud & $Y$ & $Y$ & May or may not \\
Mostly used with wireless access & May or may not & $Y$ & $Y$ \\
Focus on-line analytics & May or may not & $\mathrm{N}$ & $Y$ \\
Located between DC and device & $Y$ but can directly run on a device & $Y$ & $Y$ \\
Improve User Experience & $Y$ & $Y$ & $Y$ \\
N-tier & $N=3$ & $\mathrm{~N}=3$ or more & $\mathrm{N}=2$ or 3 \\
\hline
\end{tabular}

$Y=$ Yes; $N=$ No

solution and is optimal for latency sensitive applications but has high profiling overhead. VM based cloudlets with one-hop access to improve the response time of applications is presented in [66]. Two types of algorithms, ALL and K-step are proposed in [67] to improve the static and dynamic partitioning of cloud applications. It provides an optimal and transparent solution for distribution of different application modules and significantly improves the performance of cloud applications but it is not highly flexible yet. A framework is proposed in [68], to execute the mobile applications on the cloud virtualization environment where the user can control the deployment and execution of the application. Cloudlet Aided Cooperative Terminals Service Environment (CACTSE) is proposed in [69] for mobile content delivery service where Mobile terminals are connected with each other via Service Manager (SM) which acts like a cloudlet module to improve the user experience. Cloudlet based dictionary for mobile devices is proposed in [70] with support for translation of 6 languages. It is easily configurable and extensible but requires high processing power for fast computation. The work presented in [37], offers a dynamic cloudlets concepts. A virtual mobile cloud computing provider proposed in [71] is a resource friendly architecture. To reduce the computational workload on smartphones, mirroring approach is proposed in [59] that takes a mirror against each smartphone and virtually expand smartphones resources. COMET (Code Offload by Migrating Execution Transparently), a runtime offloading environment towards augmenting smartphones is proposed in [72] to improve computation speed but it consumes more bandwidth. A framework is proposed in [73], to support seamless mobile cloud applications execution that significantly reduces latency and power consumption. Cuckoo, a dynamic runtime system for computation offloading [74] is suitable for compute intensive operations. MOCHA (Mobile Cloud Hybrid Architecture) with mobile-cloudlet-cloud architecture is proposed in [75] for real time face recognition that gives the minimum response time. AIOLOS, a mobile middleware is proposed in [76] which improves the mobile application performance via cyber foraging and optimize execution time and energy consumption. To enable the seamless and transparent usage of cloud resources, an elastic application platform is proposed in [77] that will augment the computing capabilities of mobile devices and provides elasticity between cloud and resource constrained devices. ThinkAir is an ondemand resource allocation framework with dynamic scaling [78] where users can migrate mobile applications to the cloud and it optimizes execution time and energy consumption. Pocket Cloudlets is proposed in [79] that replicates the search and advertisement based on personalized user behaviour and improve mobile user experience. Misco, a MapReduce framework is proposed in [80] for mobile devices. It supports any device with network connectivity and support for python. XMPP-based architecture is proposed for dynamic partitioning of mobile applications deployment between cloud and mobile devices and it offers flexible and extensible architecture [81]. Mobile Augmentation Cloud Services (MACS) middleware is presented in [82] which enables adaptive application partitioning of Android services and computation offloading. It reduces local execution time. A lightweight secure cyber foraging is implemented in [83] which are useful for resource, constrained devices. It enables new applications without a new hardware investment. Cloudlet based network is proposed in [84], It considers the impact of cloudlet in interactive mobile cloud computing applications and reduces data 
transfer delay. Later, further comparisons of existing frameworks based on different parameters are given in Tables 2, 3, 4, 5 .

\section{Open issues and challenges}

In this section, some issues and challenges are highlighted that are provided direction to researchers for further research in this area.

\section{Standard protocol}

MEC being a recent technology is evolving through the phases of implementation and requires standardization emanating from collaboration of industry and researchers over an agreed platform [85].

\section{Efficient deployment}

Minimizing the latencies through optimal utilization of bandwidth may be achieved with efficient deployment of MEC. However, it is difficult to optimize the spectrum usage with dependence on complex system components.

\section{User mobility and transparency}

Provision of uninterrupted services to a frequently "onthe-move" client is another challenge in MEC environment with transparent process migration and platform heterogeneity.

\section{Heterogeneity and scalability}

As edge devices uses different access technologies including 3G, 4G, 5G, Wi-Fi and Wi-Max so aspect of heterogeneity should be catered in smooth functioning of MEC operations. This further necessitates the provision of scalability for different platforms with varying number of users $[60,62,65,67,68,71]$.

\section{Availability and security}

The availability of resources is mostly dependent upon server capacity and wireless access medium for ensuring constant service delivery. Along availability, security of data and applications from any intruder should be catered with physical measures.

Table 2 Comparison of existing frameworks part-1

\begin{tabular}{|c|c|c|c|c|c|c|c|c|c|}
\hline Properties & [60] & [61] & {$[62]$} & [63] & [64] & [65] & [66] & {$[67]$} & [68] \\
\hline Improve execution cost & Y & $N$ & $N$ & $N$ & $N$ & $N$ & $N$ & $N$ & $N$ \\
\hline Minimum execution time & N/A & $N / A$ & N/A & N/A & N/A & $N / A$ & Y & $N$ & $N$ \\
\hline Power consumption & L & L & L & L & L & L & L & L & L \\
\hline Maximum resource utilization & N/A & Y & N/A & N/A & N/A & $N / A$ & $N$ & $N$ & $N$ \\
\hline Caching support & $N / A$ & N/A & N/A & $N$ & $N$ & $N$ & Y & N/A & N/A \\
\hline Scalability & $N$ & Y & $N$ & Y & Y & $N$ & Y & $N$ & $N$ \\
\hline Complexity & N/A & N/A & N/A & L & $H$ & L & L & L & N/A \\
\hline Augmentation of resource transparency & $N$ & $N$ & $N$ & $N$ & Y & $N$ & N/A & N/A & N/A \\
\hline Programmer support & $N$ & $N$ & Y & N/A & N/A & Y & N/A & $N$ & Y \\
\hline Parallel execution support & N/A & N/A & N/A & $N$ & $N$ & $N$ & $N$ & Y & N/A \\
\hline Maximum throughput & N & Y & $N$ & N & $N$ & $N$ & N/A & N/A & N/A \\
\hline Network latency & L & $N$ & L & L & L & L & L & L & $H$ \\
\hline Optimize bandwidth utilization & N/A & $N / A$ & N/A & $N / A$ & N/A & $N / A$ & $N$ & $N$ & $N$ \\
\hline QoS & N/A & $N$ & Y & $N$ & $N / A$ & $N$ & N/A & N/A & Y \\
\hline Guaranteed Bandwidth & N/A & N/A & N/A & $N$ & $N$ & $N$ & $N$ & N/A & Y \\
\hline Network Load & N/A & $N / A$ & N/A & $H$ & L & L & L & L & N/A \\
\hline Transmission delay & L & $H$ & $H$ & N/A & N/A & L & N/A & M & L \\
\hline Reduction in number of hops & N/A & N/A & N/A & Y & Y & Y & Y & N/A & $N$ \\
\hline Security overhead & M & N/A & N/A & N/A & M & $N / A$ & M & N/A & $H$ \\
\hline Fault tolerance & N & N & Y & $N / A$ & N/A & Y & N/A & N & Y \\
\hline Pre-execution delay & N/A & $N / A$ & $N / A$ & $H$ & $H$ & $H$ & M & N/A & N/A \\
\hline Usage of high bandwidth links & N/A & N/A & N/A & Y & Y & Y & Y & N/A & Y \\
\hline Reduce of floading & N/A & N/A & N/A & $N / A$ & N/A & $N / A$ & $N$ & $N$ & Y \\
\hline time Optimize data transfer cost & $N / A$ & N/A & N/A & N/A & $N / A$ & $N / A$ & Y & Y & Y \\
\hline
\end{tabular}

$Y=$ Yes; $N=$ No; $H=$ High; $M=$ Medium, $L=$ Low; $N / A=$ Not applicable 
Table 3 Comparison of existing frameworks part-2

\begin{tabular}{|c|c|c|c|c|c|c|c|c|c|}
\hline Properties & {$[60]$} & [61] & {$[62]$} & {$[63]$} & [64] & {$[65]$} & {$[66]$} & {$[67]$} & [68] \\
\hline Data transfer overhead & $H$ & $H$ & $H$ & $N / A$ & $H$ & L & L & $H$ & $M$ \\
\hline VM migration overhead & $H$ & N/A & $N / A$ & $N / A$ & $H$ & $N / A$ & L & $N / A$ & M \\
\hline Optimize deployment & $N$ & $N$ & Y & $N$ & $N$ & $N$ & $N / A$ & $N / A$ & $N / A$ \\
\hline Profiler Overhead & $H$ & M & L & $N / A$ & L & $H$ & $N / A$ & $H$ & L \\
\hline Cloud usage overhead & $H$ & M & L & L & $H$ & L & M & L & L \\
\hline Operational cost & $H$ & L & L & M & $H$ & L & M & L & $L$ \\
\hline Deploys mirror & N/A & $N / A$ & $N / A$ & $N$ & $N$ & $N$ & $N$ & $N / A$ & $N / A$ \\
\hline Partitioning overhead & L & L & $H$ & $N / A$ & N/A & M & $N / A$ & M & M \\
\hline Offloading overhead & $H$ & $H$ & $H$ & $N / A$ & N/A & $H$ & $N / A$ & $H$ & $H$ \\
\hline Method call overhead & $H$ & $H$ & $H$ & N/A & $N / A$ & $H$ & $N / A$ & $H$ & $H$ \\
\hline
\end{tabular}

$Y=$ Yes; $N=$ No; $H=$ High; $M=$ Medium; $L=$ Low $N / A=$ Not applicable

\section{Fog-cloud interworking}

When considering connectivity challenges for Gateways and/or Fog nodes, there are three different aspects to consider in any end-to-end system:

- Northbound connections, which are the connections between Gateways/Fog nodes and a Cloud service (public or private).

- Southbound connections, which are the connections between the Gateway/Fog node and the Edge devices/ things/sensor networks.
- East/West connections, which are the connections between Gateways/Fog nodes themselves, so that they can share data without requiring, Cloud connectivity.

\section{Data management}

The data management capabilities required include (but are not limited to):

- Data normalization, which is ingesting, aligning and enriching the data from different sources (Things,

Table 4 Comparison of existing frameworks part-3

\begin{tabular}{|c|c|c|c|c|c|c|c|c|c|}
\hline Properties & [69] & [70] & {$[37]$} & [71] & [59] & [72] & [73] & [74] & [75] \\
\hline Improve execution cost & $N$ & $N$ & $N$ & $N$ & Y & $N$ & $N$ & $N$ & $N$ \\
\hline Minimum execution time & $N$ & $N$ & $N$ & $N$ & $N$ & Y & $N$ & Y & $N$ \\
\hline Power consumption & L & L & L & $L$ & L & L & L & L & M \\
\hline Maximum resource utilization & $N$ & $N$ & Y & Y & $N$ & $N$ & $N$ & $N$ & $N$ \\
\hline Caching support & N/A & N/A & $N$ & $N$ & Y & Y & $N / A$ & $N / A$ & $N / A$ \\
\hline Scalability & Y & Y & Y & $N$ & Y & Y & Y & Y & Y \\
\hline Programmer support & $N$ & N/A & Y & $N$ & N/A & L & N/A & N/A & $N / A$ \\
\hline Parallel execution support & $N / A$ & $N / A$ & $N$ & $N$ & $N$ & Y & N/A & $N / A$ & $N / A$ \\
\hline Network latency & L & L & $L$ & L & L & L & L & L & $H$ \\
\hline Optimize bandwidth utilization & Y & $N$ & $N$ & $N$ & $N$ & $N$ & $N$ & $N$ & $N$ \\
\hline QoS & Y & N/A & Y & $N$ & N/A & N/A & $N$ & N/A & Y \\
\hline Minimum response time & $N$ & $N$ & $N$ & $N$ & $N$ & $N$ & $N$ & $N$ & Y \\
\hline Guaranteed Bandwidth & $N / A$ & N/A & $N$ & N & $N$ & $N$ & N/A & $N / A$ & $N / A$ \\
\hline Reduction in number of hops & $N / A$ & N/A & Y & Y & Y & Y & $N / A$ & $N / A$ & $N / A$ \\
\hline Pre-execution delay & $N / A$ & N/A & M & $H$ & L & $L$ & N/A & $N / A$ & $N / A$ \\
\hline Usage of high bandwidth links & N/A & $N / A$ & Y & Y & $N$ & Y & N/A & $N / A$ & $N / A$ \\
\hline Profiler Overhead & $N / A$ & $N / A$ & $H$ & N/A & N/A & $N / A$ & Y & $N / A$ & $N / A$ \\
\hline Cloud usage overhead & L & M & L & $H$ & $H$ & L & $H$ & L & M \\
\hline Operational cost & L & L & L & L & $H$ & L & $H$ & L & $H$ \\
\hline Deploys mirror & N/A & N/A & $N$ & $N$ & $Y$ & $N$ & N/A & N/A & $N / A$ \\
\hline
\end{tabular}

$Y=$ Yes; $N=$ No; $H=$ High; $M=$ Medium; $L=$ low; $N / A=$ Not applicable 
Table 5 Comparison of existing frameworks part-4

\begin{tabular}{|c|c|c|c|c|c|c|c|c|c|}
\hline Properties & [76] & [77] & {$[78]$} & [79] & [80] & [81] & [82] & [83] & {$[84]$} \\
\hline Improve execution cost & $N$ & $N$ & Y & $N$ & $N$ & $N$ & Y & N/A & N/A \\
\hline Minimum execution time & Y & N & Y & N & N & $N$ & N & $N / A$ & N/A \\
\hline Power consumption & L & L & L & L & $H$ & L & L & N/A & N/A \\
\hline Caching support & Y & N/A & $N$ & Y & N/A & N/A & Y & Y & Y \\
\hline Scalability & $N$ & Y & Y & Y & Y & Y & N & N/A & N/A \\
\hline Programmer support & $N$ & Y & Y & N/A & N/A & Y & Y & N/A & N/A \\
\hline Parallel execution support & N/A & N/A & Y & N & N/A & N/A & $N$ & $N$ & N \\
\hline Maximum cache hit rate & N/A & N & $N$ & Y & $N$ & $N$ & $N$ & N/A & N/A \\
\hline Minimum missed deadlines & N/A & $N$ & $N$ & $N$ & Y & $N$ & N & N/A & N/A \\
\hline Network latency & L & $H$ & N/A & L & L & M & L & N/A & N/A \\
\hline Qos & N & Y & $N$ & N & N & Y & N & N/A & N/A \\
\hline Guaranteed Bandwidth & $N$ & N/A & $N$ & $N$ & N/A & N/A & $N$ & $N$ & N \\
\hline Transmission delay & M & $H$ & $H$ & N/A & N/A & M & M & N/A & N/A \\
\hline Reduction in number of hops & $N$ & N/A & $N$ & Y & N/A & N/A & Y & Y & Y \\
\hline Fault tolerance & Y & Y & Y & N/A & N/A & N & N & N/A & N/A \\
\hline Pre-execution delay & $H$ & N/A & $H$ & L & N/A & N/A & $H$ & L & $H$ \\
\hline Usage of high bandwidth links & Y & N/A & Y & Y & N/A & N/A & Y & Y & Y \\
\hline Maximum privacy and security & N/A & $N$ & $N$ & $N$ & $N$ & Y & Y & N/A & N/A \\
\hline Maximum throughput & N/A & Y & $N$ & $N$ & $N$ & $N$ & $N$ & N/A & Y \\
\hline Data transfer overhead & $H$ & L & $H$ & N/A & N/A & $H$ & L & $H$ & N/A \\
\hline Profiler Overhead & L & $H$ & $H$ & M & $H$ & $H$ & $H$ & N/A & N/A \\
\hline Cloud usage overhead & L & $H$ & $H$ & L & L & L & L & $H$ & $H$ \\
\hline Operational cost & L & $H$ & L & L & L & M & L & N/A & N/A \\
\hline Deploys mirror & N & N/A & N & N & N/A & N/A & N & N & $N$ \\
\hline Partitioning overhead & $H$ & $H$ & $H$ & N/A & N/A & $H$ & L & N/A & N/A \\
\hline Offloading overhead & $H$ & $H$ & $H$ & N/A & N/A & L & $H$ & N/A & N/A \\
\hline Method call overhead & L & $H$ & $H$ & $N / A$ & $N / A$ & $H$ & $H$ & $N / A$ & N/A \\
\hline
\end{tabular}

$Y=$ Yes; $N=$ No; $H=$ High; $M=$ Medium; $L=$ Low; $N / A=$ Not applicable

devices and sensors) into a common data model with well understood semantics.

- Filtering and querying data, so that applications and analytics can efficiently access and use the data relevant to them.

- Integration with Edge analytics, because the whole reason for capturing these data is to be able to analyze them, create new actionable insights, make decisions and put those decisions into action. Transforming data into different representations and formats, for the purposes of integrating with the IoT ecosystem.

- Aggregating data and/or abstract meta-data, as preparation for local analytics or pushing it to Cloud services.

\section{Conclusion}

The paper presents a comprehensive review of the prevalent MEC frameworks along with a comparative analysis of contemporary approaches with respects to different performance parameters. Comparative analysis employs different parameters such as such as system performance, network performance, overhead of deployment and system migration overhead to measure the degree of effectiveness of different approaches. Based on our thorough investigation, it can be asserted that MEC is a way forward for achieving $1 \mathrm{~ms}$ latency dream. Therefore, researcher has proposed several MEC architecture to reduce the latency. While considering the state-of-the-art presented in this paper, many areas are still open for further research to investigate a comprehensive architecture design with intelligent migration mechanism for multi-access mobile edge computing.

\section{Abbreviations}

CACTSE: Cloudlet Aided Cooperative Terminals Service Environment; CC: Cloud Computing; COMET: Code Offload by Migrating Execution Transparently; EdgeC: Edge Cloud; eNB: Evolved Node B; loE: Internet of 
Everything; loM: Internet of Me; loT: Internet of Things; MACS: Mobile Augmentation Cloud Services; MCC: Mobile Cloud Computing; MEC: Mobile Edge Computing; MOCHA: Mobile Cloud Hybrid Architecture; OEC: Open Edge Computing; PEFC: Performance Enhancement Framework for Cloudlet; QoE: Quality of Experience; QoS: Quality of Service; RAN: Radio Access Network; SDN: Software Defined Networking; SM: Service Manager; V2X: Vehicle-to-everything

\section{Acknowledgments}

We are thankful to the Sohail Sarwar for proofreading the paper to improve the write up of the manuscript.

The present work was undertaken in the context of the "SelfOrganizatioN toward reduced cost and eNergy per bit for future Emerging radio Technologies" with contract number 734545. The project has received research funding from the H2020-MSCA-RISE-2016 European Framework Program.

\section{Funding}

The present work was undertaken in the context of the "SelfOrganizatioN toward reduced cost and eNergy per bit for future Emerging radio Technologies" with contract number 734545. The project has received research funding from the H2020-MSCA-RISE-2016 European Framework Program.

\section{Availability of data and materials}

Not applicable.

\section{Authors' contributions}

SS contributed to Cloudlet approach and Fog Consortium, classification and detail comparison of existing frameworks. MI has been involved in drafting the manuscript and revising it critically during initial and revised submissions. MI also contributed to the open issues and challenges. TD contributed to Edge Cloud Computing Standardization and Fora with a detailed comparison of edge cloud approaches including MEC approach. ZQ contributed to the introduction part. All authors read and approved the final manuscript.

\section{Authors' information}

Sonia Shahzadi received her BS and MS degrees from University of Gujrat, Pakistan, in 2013 and 2016 respectively. She is currently associated with Swan Mesh Networks Ltd., Research and Development, London, UK. Her research interests include Cloud Computing and Mobile Edge Computing. Muddesar labal is Senior Lecturer in Mobile Computing in the Division of Computer Science and Informatics, School of Engineering. He won an EPSRC Doctoral Training Award in 2007 and completed his PhD from Kingston University in 2010 with a dissertation titled "Design, development, and implementation of a high-performance wireless mesh network for application in emergency and disaster recovery". He has been a principal investigator, co-investigator, project manager, coordinator and focal person of more than 10 internationally teamed research and development, capacity building and training projects. He is an established researcher and expert in the fields of: mobile cloud computing and open-based networking for applications in Education, disaster management and healthcare; community networks; and smart cities. His research interests include 5G networking technologies, multimedia cloud computing, mobile edge computing, fog computing, Internet of Things, software-defined networking, network function virtualization, quality of experience, and cloud infrastructures and services. Tasos Dagiuklas is a leading researcher and expert in the fields of Internet and multimedia technologies for smart cities, ambient assisted living, healthcare and smart agriculture. He is the leader of the SuITE research group at the London South Bank University where he also acts as the Head of Division in Computer Science. Tasos Dagiuklas received the Engineering Degree from the University of Patras-Greece in 1989, the M.Sc. from the University of Manchester-UK in 1991 and the Ph.D. from the University of Essex-UK in 1995, all in Electrical Engineering. He has been a principle investigator, co-investigator, project and technical manager, coordinator and focal person of more than 20 internationally R\&D and Capacity training projects with total funding of approximately $£ 5.0 \mathrm{~m}$ from different international organizations. His research interests include Smart Internet Technologies, Media Optimization across heterogeneous networks, QoE, Virtual Reality, Augmented Reality and cloud infrastructures and services. Zia UI Qayyum is currently working as a vice chancellor at National University of Gujrat, Pakistan. He has over 20 years of experience in teaching and research. His major areas of interest include Data Mining, Knowledge Representation, Information Retrieval and Emerging Communication Technologies.

\section{Competing interests}

The authors declare that they have no competing interests.

\section{Publisher's Note}

Springer Nature remains neutral with regard to jurisdictional claims in published maps and institutional affiliations.

\section{Author details}

'Swan Mesh Networks Ltd, London, UK. ${ }^{2}$ London South Bank University, London, UK. ${ }^{3}$ University of Gujrat, Gujrat, Pakistan.

Received: 27 April 2017 Accepted: 20 November 2017 Published online: 21 December 2017

\section{References}

1. Internet of Everything. http://www.cisco.com/c/dam/en_us/about/ac79/ docs/innov/loE_Economy.pdf. Accessed 17 July 2017

2. RCRWireless. http://www.rcrwireless.com/20160628/opinion/reality-check50b-iot-devices-connected-2020-beyond-hype-reality-tag10. Accessed 17 July 2017

3. ETSI Mobile Edge Computing Portal. http://www.etsi.org/technologiesclusters/technologies/mobile-edge-computing. Accessed 27 Jan 2016

4. Patel M, Naughton B, Chan C, Sprecher N, Abeta S, Neal, et al. Mobile-edge computing introductory technical white paper. White paper, mobile-edge computing (MEC) industry initiative (2014)

5. Second white paper on Mobile Edge Computing from ETSI. http://www.etsi. org/images/files/ETSIWhitePapers/etsi_wp11_mec_a_key_technology_ towards_5g.pdf. Accessed 27 Jan 2016

6. ITU-T report about Tactile Internet, Aug 2014. https://www.itu.int/dms_pub/ itu-t/oth/23/01/T23010000230001PDFE.pdf. Accessed 27 Jan 2016

7. Victor Bahl, Microsoft, 13 May 2015. http://research.microsoft.com/en-us/ um/people/bahl/Present/Bahl_mDC_Keynote_May.pdf. Accessed 27 Jan 2016

8. Vaquero $L M$, Rodero-Merino $L$ (2014) Finding your way in the fog: towards a comprehensive definition of fog computing. ACM SIGCOMM Comput Commun Rev 44(5):27-32

9. Li K-C, Li Q, Shih TK (2014) Cloud computing and digital media: fundamentals, techniques, and applications. CRC Press

10. Tran TX, Hajisami A, Pandey P, Pompili D (2017) Collaborative mobile edge computing in $5 \mathrm{~g}$ networks: new paradigms, scenarios, and challenges. IEEE Commun Mag 55(4):54-61

11. Wang X, Han G, Du X, Rodrigues JJ (2015) Mobile cloud computing in 5g: emerging trends, issues, and challenges [guest editorial]. IEEE Netw 29(2):4-5

12. Ahmed E, Rehmani MH (2017) Mobile edge computing: opportunities, solutions, and challenges. Elsevier

13. Clinch S, Harkes J, Friday A, Davies N, Satyanarayanan M (2012) How close is close enough? Understanding the role of cloudlets in supporting display appropriation by mobile users. In: Pervasive computing and communications (PerCom), 2012 IEEE international conference on. IEEE, pp 122-127

14. Zhuang W (2017) Emerging technologies, applications, and standardizations for connecting vehicles (part ii) [from the guest editor]. IEEE Veh Technol Mag 12(2):23-25

15. Zhang $K$, Mao $Y$, Leng $S$, He Y, ZHANG Y (2017) Predictive offloading in cloud-driven vehicles: using mobile-edge computing for a promising network paradigm. IEEE Veh Technol Mag 12:36-44

16. Gubbi J, Buyya R, Marusic S, Palaniswami M (2013) Internet of things (iot): a vision, architectural elements, and future directions. Futur Gener Comput Syst 29(7):1645-1660

17. Chen S, Xu H, Liu D, Hu B, Wang H (2014) A vision of iot: applications, challenges, and opportunities with china perspective. IEEE Internet Things J 1(4):349-359

18. Botta, A., De Donato, W., Persico, V., Pescap'e, A. Pescap'e, A: On the integration of cloud computing and internet of things. In: Future internet of things and cloud (FiCloud), 2014 international conference on, pp. 23-30 (2014). IEEE

19. Corcoran P, Datta SK (2016) Mobile-edge computing and the internet of things for consumers: extending cloud computing and services to the edge of the network. IEEE Consum Electron Mag 5(4):73-74

20. Dastjerdi AV, Buyya R (2016) Fog computing: helping the internet of things realize its potential. Computer 49(8):112-116 
21. Chen S, Wang Y, Pedram M (2013) A semi-markovian decision process based control method for offloading tasks from mobile devices to the cloud. In: Global communications conference (GLOBECOM), 2013 IEEE. IEEE, pp 2885-2890

22. Beck MT, Werner M, Feld S, Schimper T. Mobile edge computing: a taxonomy (2014)

23. Beck MT, Maier M. Mobile edge computing: challenges for future virtual network embedding algorithms (2014)

24. Chen X, Jiao L, Li W, Fu X. Efficient multi-user computation offloading for mobile-edge cloud computing (2015)

25. Grieco R, Malandrino D, Scarano V (2006) A scalable cluster-based infrastructure for edge-computing services. World Wide Web 9(3):317-341

26. Dey S, Mukherjee A, Paul HS, Pal A (2013) Challenges of using edge devices in iot computation grids. In: Parallel and distributed systems (ICPADS), 2013 international conference on. IEEE, pp 564-569

27. Desertot M, Escoffier C, Lalanda P, Donsez D (2006) Autonomic management of edge servers. In: Self-Organizing Systems. Springer, pp 216-229

28. Zhu W, Luo C, Wang J, Li S (2011) Multimedia cloud computing. IEEE Signal Process Mag 28(3):59-69

29. Machen A, Wang S, Leung KK, Ko BJ, Salonidis T (2016) Migrating running applications across mobile edge clouds: poster. In: Proceedingsof the 22nd annual international conference on mobile computing and networking. ACM, pp 435-436

30. Li H, Shou G, Hu Y, Guo Z (2016) Mobile edge computing: progress and challenges. In: Mobile cloud computing, services, and engineering (MobileCloud), 2016 4th IEEE international conference on. IEEE, pp 83-84

31. Ahmed E, Gani A, Sookhak M, Ab Hamid SH, Xia F (2015) Application optimization in mobile cloud computing: motivation, taxonomies, and open challenges. J Netw Comput Appl 52:52-68

32. Ahmed E, Gani A, Khan MK, Buyya R, Khan SU (2015) Seamless application execution in mobile cloud computing: motivation, taxonomy, and open challenges. J Netw Comput Appl 52:154-172

33. Elijah: Cloudlet-based Edge Computing. http://elijah.cs.cmu.edu/. Accessed 19 July 2017

34. Khan KA, Wang Q, Luo C, Wang X, Grecos C (2014) Comparative study of internet cloud and cloudlet over wireless mesh networks for real-time applications. In: SPIE Photonics Europe. International Society for Optics and Photonics, pp 91390-91390

35. OpenEdgeComputing. http://openedgecomputing.org/. Accessed 19 July 2017

36. Khan KA, Wang Q, Grecos C (2012) Experimental framework of integrated cloudlets and wireless mesh networks. In: Telecommunications forum (TELFOR), 2012 20th. IEEE, pp 190-193

37. Verbelen T, Simoens P, De Turck F, Dhoedt B (2012) Cloudlets: bringing the cloud to the mobile user. In: Proceedings of the third ACM workshop on mobile cloud computing and services. ACM, pp 29-36

38. Jararweh Y, Tawalbeh L, Ababneh F, Dosari F (2013) Resource efficient mobile computing using cloudlet infrastructure. In: Mobile ad-hoc and sensor networks (MSN), 2013 IEEE ninth international conference on. IEEE, pp 373-377

39. Whaiduzzaman M, Gani A, Naveed A (2014) Pefc: performance enhancement framework for cloudlet in mobile cloud computing. In: Robotics and manufacturing automation (ROMA), 2014 IEEE international symposium on. IEEE, pp 224-229

40. Routaib H, Badidi E, Elmachkour M, Sabir E, Elkoutbi M (2014) Modeling and evaluating a cloudlet-based architecture for mobile cloud computing. In: Intelligent systems: theories and applications (SITA-14), 2014 9th international conference on. IEEE, pp 1-7

41. Li Y, Wang W (2013) The unheralded power of cloudlet computing in the vicinity of mobile devices. In: Global Communications Conference (GLOBECOM), 2013 IEEE. IEEE, pp 4994-4999

42. Ceselli A, Premoli M, Secci S (2015) Cloudlet network design optimization. In: Proc. of IFIP networking

43. Rawadi J, Artail H, Safa H (2014) Providing local cloud services to mobile devices with inter-cloudlet communication. In: Mediterranean Electrotechnical conference (MELECON), 2014 17th IEEE. IEEE, pp 134-138

44. Jararweh $\mathrm{Y}$, Doulat $\mathrm{A}$, AlQudah $\mathrm{O}$, Ahmed E, Al-Ayyoub M, Benkhelifa E (2016) The future of mobile cloud computing: integrating cloudlets and mobile edge computing. In: Telecommunications (ICT), 2016 23rd international conference on. IEEE, pp 1-5

45. Niyato D, Wang P, Joo PCH, Han Z, Kim DI (2014) Optimal energy management policy of a mobile cloudlet with wireless energy charging. In:
Smart grid communications (SmartGridComm), 2014 IEEE international conference on. IEEE, pp 728-733

46. tactical-cloudlets. https://www.govtechworks.com/tactical-cloudlets-mobilecomputing-readies-for-battle/\#gs.T=_DGTc. Accessed 22 July 2017

47. Lewis G, Echeverr'ı S, Simanta S, Bradshaw B, Root J (2014) Tactical cloudlets: moving cloud computing to the edge. In: Military communications conference (MILCOM), 2014 IEEE. IEEE, pp 1440-1446

48. Liu Y, Lee MJ, Zheng Y (2016) Adaptive multi-resource allocation for cloudlet-based mobile cloud computing system. IEEE Trans Mob Comput 15(10):2398-2410

49. Stojmenovic I, Wen S (2014) The fog computing paradigm: scenarios and security issues. In: Computer science and information systems (FedCSIS), 2014 federated conference on. IEEE, pp 1-8

50. Bonomi F, Milito R, Zhu J, Addepalli S (2012) Fog computing and its role in the internet of things. In: Proceedings of the first edition of the MCC workshop on mobile cloud computing. ACM, pp 13-16

51. Shenoy K, Bhokare P, Pai U (2015) Fog computing future of cloud computing. Int J Sci Res 4(6):55-56

52. Suryawanshi R, Mandlik G. Focusing on mobile users at edge and internet of things using fog computing (2015)

53. Aazam M, Huh E-N (2014) Fog computing and smart gateway based communication for cloud of things. In: Future internet of things and cloud (FiCloud), 2014 international conference on. IEEE, pp 464-470

54. Bonomi F, Milito R, Natarajan P, Zhu J (2014) Fog computing: a platform for internet of things and analytics. In: Big data and internet of things: a roadmap for smart environments. Springer, pp 169-186

55. Luan TH, Gao L, Li Z, Xiang Y, Sun L (2015) Fog computing: focusing on mobile users at the edge, p. 1-11. arXiv:1502.01815v3. https://arxiv.org/abs/ 1502.01815

56. Peng M, Yan S, Zhang K, Wang C. Fog computing based radio access networks: issues and challenges. arXiv preprint arXiv:1506.04233 (2015)

57. Firdhous M, Ghazali O, Hassan S (2014) Fog computing: will it be the future of cloud computing? In: Third international conference on Informatics \& Applications, Kuala Terengganu, Malaysia, pp 8-15

58. Truong NB, Lee GM, Ghamri-Doudane Y (2015) Software defined networkingbased vehicular Adhoc Network with Fog Computing. Integrated Network Management (IM), 2015 IFIP/IEEE International Symposium on, p. 1202-1207. http://ieeexplore.ieee.org/abstract/document/7140467/

59. Zhao B, Xu Z, Chi C, Zhu S, Cao G (2012) Mirroring smartphones for good: a feasibility study. In: Mobile and ubiquitous systems: computing, networking, and services. Springer, pp 26-38

60. Chun B-G, Ihm S, Maniatis P, Naik M, Patti A (2011) Clonecloud: elastic execution between mobile device and cloud. In: Proceedings of the sixth conference on computer systems. ACM, pp 301-314

61. Yang L, Cao J, Yuan Y, Li T, Han A, Chan A (2013) A framework for partitioning and execution of data stream applications in mobile cloud computing. ACM Sigmetrics Perform Eval Rev 40(4):23-32

62. Verbelen T, Stevens T, Simoens P, De Turck F, Dhoedt B (2011) Dynamic deployment and quality adaptation for mobile augmented reality applications. J Syst Softw 84(11):1871-1882

63. Marinelli EE. Hyrax: cloud computing on mobile devices using mapreduce. Technical report, DTIC document (2009)

64. Chun B-G, Maniatis P (2009) Augmented smartphone applications through clone cloud execution. In: HotOS, vol 9, pp 8-11

65. Cuervo E, Balasubramanian A, Cho D-K, Wolman A, Saroiu S, Chandra R, Bahl P (2010) Maui: making smartphones last longer with code offload. In: Proceedings of the 8th international conference on mobile systems, applications, and services. ACM, pp 49-62

66. Satyanarayanan M, Bahl P, Caceres R, Davies N (2009) The case for vm-based cloudlets in mobile computing. IEEE Pervasive Comput 8(4):14-23

67. Giurgiu I, Riva O, Juric D, Krivulev I, Alonso G (2009) Calling the cloud: enabling mobile phones as interfaces to cloud applications. In: Middleware 2009. Springer, pp 83-102

68. Hung S-H, Shih C-S, Shieh J-P, Lee C-P, Huang Y-H (2012) Executing mobile applications on the cloud: framework and issues. Comput Math Appl 63(2):573-587

69. Qing W, Zheng H, Ming W, Haifeng L (2013) Cactse: cloudlet aided cooperative terminals service environment for mobile proximity content delivery. China Commun 10(6):47-59

70. Achanta VS, Sureshbabu NT, Thomas V, Sahitya ML, Rao S (2012) Cloudletbased multi-lingual dictionaries. In: Services in Emerging Markets (ICSEM), 2012 third international conference on. IEEE, pp 30-36 
71. Huerta-Canepa G, Lee D (2010) A virtual cloud computing provider for mobile devices. In: Proceedings of the 1st ACM workshop on Mobile Cloud Computing \& Services: social networks and beyond. ACM, p 6

72. Gordon MS, Jamshidi DA, Mahlke SA, Mao ZM, Chen X (2012) Comet: code offload by migrating execution transparently. In: OSDI, pp 93-106

73. Lee B-D (2012) A framework for seamless execution of mobile applications in the cloud. In: Recent advances in computer science and information engineering. Springer, pp 145-153

74. Kemp R, Palmer N, Kielmann T, Bal HE (2010) Cuckoo: a computation offloading framework for smartphones. In: MobiCASE. Springer, pp 59-79

75. Soyata T, Muraleedharan R, Funai C, Kwon M, Heinzelman W (2012) Cloudvision: real-time face recognition using a mobile-cloudlet-cloud acceleration architecture. In: Computers and communications (ISCC), 2012 IEEE symposium on. IEEE, pp 000059-000066

76. Verbelen T, Simoens P, De Turck F, Dhoedt B (2012) Aiolos: middleware for improving mobile application performance through cyber foraging. I Syst Softw 85(11):2629-2639

77. Zhang X, Jeong S, Kunjithapatham A, Gibbs S (2010) Towards an elastic application model for augmenting computing capabilities of mobile platforms. In: Mobile wireless middleware, operating systems, and applications. Springer, pp 161-174

78. Kosta S, Aucinas A, Hui P, Mortier R, Zhang X (2012) Thinkair: dynamic resource allocation and parallel execution in the cloud for mobile code offloading. In: INFOCOM, 2012 proceedings IEEE. IEEE, pp 945-953

79. Koukoumidis E, Lymberopoulos D, Strauss K, Liu J, Burger D (2011) Pocket cloudlets. ACM SIGARCH computer architecture news. ASPLOS XVI Proceedings of the sixteenth international conference on Architectural support for programming languages and operating systems 39(1):171-184. https://dl.acm.org/citation.cfm?id=1950387

80. Dou A, Kalogeraki V, Gunopulos D, Mielikainen T, Tuulos VH (2010) Misco: a mapreduce framework for mobile systems. In: Proceedings of the 3rd international conference on pervasive technologies related to assistive environments. ACM, p 32

81. Kovachev D, Cao Y, Klamma R (2010) Augmenting pervasive environments with an xmpp-based mobile cloud middleware. In: Mobile computing, applications, and services. Springer, pp 361-372

82. Kovachev D, Yu T, Klamma R (2012) Adaptive computation offloading from mobile devices into the cloud. In: Parallel and distributed processing with applications (ISPA), 2012 IEEE 10th international symposium on. IEEE, pp 784-791

83. Goyal S, Carter J (2004) A lightweight secure cyber foraging infrastructure for resource-constrained devices. In: Mobile computing systems and applications, 2004. WMCSA 2004. Sixth IEEE workshop on. IEEE, pp 186-195

84. Fesehaye D, Gao Y, Nahrstedt K, Wang G (2012) Impact of cloudlets on interactive mobile cloud applications. In: Enterprise distributed object computing conference (EDOC), 2012 IEEE 16th international. IEEE, pp 123-132

85. Ahmed A, Ahmed E (2016) A survey on mobile edge computing. In: Intelligent systems and control (ISCO), 2016 10th international conference on. IEEE, pp 1-8

\section{Submit your manuscript to a SpringerOpen ${ }^{\circ}$ journal and benefit from:}

- Convenient online submission

- Rigorous peer review

Open access: articles freely available online

- High visibility within the field

- Retaining the copyright to your article 